\title{
Robotic or laparoscopic surgery for rectal cancer - which is the best answer? a comprehensive review of non-oncological outcomes and learning curve
}

\author{
Sandra L. Kavalukas, Amandeep Ghuman, Stephen P. Sharp, Steven D. Wexner \\ Department of Colorectal Surgery, Cleveland Clinic Florida, Weston, FL 33331, USA.
}

Correspondence to: Dr. Steven D. Wexner, Department of Colorectal Surgery, Cleveland Clinic Florida, Weston, FL 33331, USA. E-mail:wexners@ccf.org

\begin{abstract}
How to cite this article: Kavalukas SL, Ghuman A, Sharp SP, Wexner SD. Robotic or laparoscopic surgery for rectal cancer - which is the best answer? a comprehensive review of non-oncological outcomes and learning curve. Mini-invasive Surg 2020;4:61. http://dx.doi.org/10.20517/2574-1225.2020.71
\end{abstract}

Received: 8 Jul 2020 First Decision: 6 Aug 2020 Revised: 13 Aug 2020 Accepted: 14 Aug 2020 Published: 1 Sep 2020

Academic Editor: S. W. Larach Copy Editor: Cai-Hong Wang Production Editor: Jing Yu

\begin{abstract}
Much effort has been spent evaluating the difference between robotic and laparoscopic surgery platforms for rectal cancer. There is a plethora of literature comparing outcomes for intraoperative events, postoperative complications, long term outcomes, cost, and learning curve. The data are conclusive regarding the higher cost of robotic surgery compared to laparoscopic surgery. This article is a comprehensive review of the available literature regarding intraoperative and postoperative outcomes. For practically all parameters evaluated, there are no significant differences between the two platforms. The ultimate decision on whether to perform robotic $v s$. laparoscopic surgery should be based on surgeon preference and familiarity with equipment, as well as local resources.
\end{abstract}

Keywords: Rectal cancer, rectal carcinoma, robotic, robotics, laparoscopy, total mesorectal excision

\section{INTRODUCTION}

Surgical management of rectal cancer has undergone an impressive evolution during the past thirty years. An explosion of minimally invasive techniques has advanced almost every colorectal operation since the first reports of laparoscopic segmental and total colectomy ${ }^{[1]}$. Advantages of laparoscopic surgery compared to laparotomy include reductions in postoperative pain, length of stay, incisional hernia, adhesive

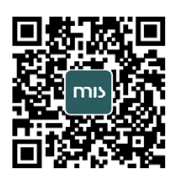


bowel obstruction, wound complications, and mortality ${ }^{[2-5]}$. The disadvantages of laparoscopic surgery comprise a relative loss of tactile sensation compared to open surgery and technical difficulty with fine movement. There is heightened awareness of surgeon ergonomics due to overuse injuries and workplace musculoskeletal disorders in laparoscopic surgeons. Improper table height, position of the monitors, and handling of long instruments are factors contributing to these afflictions.

The introduction of the robotic DaVinci operating system (Sunnyvale, CA) in 2000 brought forth another dimension of minimally invasive technology. This platform was utilized in colorectal surgery in March 2001 when the first sigmoid and right colectomies were described ${ }^{[6]}$. Robotic surgery has now gained widespread acceptance both in terms of surgeon satisfaction and patient outcomes. The strengths of robotic surgery lie principally in wristed instruments providing seven degrees of freedom. Many surgeons endorse greater comfort during the procedure, improved visualization of the operative field, and less technical difficulty operating in challenging locations including the narrow pelvis. The limitations of robotic surgery can include the operative time required to dock the robot, loss of tactile sensation, and increased cost compared to laparoscopic surgery.

Standards by which successful surgical outcomes are evaluated examine intraoperative events, postoperative complications, and long-term sequelae. These facets have been extensively studied comparing laparoscopic and robotic surgery. This paper explores any potential differences between the two methods regarding nononcological perioperative outcomes. Conversion rate, postoperative pain or ileus, anastomotic leak, surgical site infection, length of stay, cost, long-term urogenital function, and learning curve are the specific topics that are addressed.

\section{INTRAOPERATIVE OUTCOME}

\section{Conversion rate}

Challenges that present to a surgeon include patient body habitus, fibrosis from chronic inflammatory processes, adherence to surrounding structures by infiltrating tumours, and adhesions from previous surgeries. These situations can result in conversion to an open procedure. One of the unique tasks specific to colorectal surgeons is removing a low rectal tumour. Particularly in a narrow pelvis, this task can be challenging due to the limited range of motion of laparoscopic instruments. Colorectal robotic surgery first gained popularity specifically for this scenario. Precise dissection down to the pelvic floor with wristed instruments facilitates total mesorectal excision over the pelvic brim.

Given the above situations, investigators hypothesized that robotic surgery would result in a lower conversion rate compared to laparoscopic procedures, but the data exhibit many conflicting reports when examining conversion rates. One must consider the research design when interpreting these results. A few studies utilized nation-wide databases, while others performed retrospective reviews, case-matched studies, or propensity-matched groups. Several studies report equivalent conversion rates between laparoscopic and robotic procedures ${ }^{[7-10]}$. Feinberg et al. ${ }^{[11]}$ performed a retrospective National Surgical Quality Improvement Program study of over 8,864 patients undergoing either laparoscopic or robotic colorectal procedures, finding a statistically significant difference in the conversion rate of $13.7 \%$ and $9.5 \%$ for laparoscopic and robotic procedures, respectively $(P<0.008)$. A subgroup analysis was performed to identify risk factors for increased conversion rates, finding that patients with colon cancer [odds ratio (OR) 1.8], Crohn's disease (OR 2.19), and diverticular disease (OR 1.9) had higher likelihood of conversion. The two most interesting findings in this study were that neither body mass index $>30 \mathrm{~kg} / \mathrm{m}^{2}$ nor rectal resection procedures conferred a significant risk of conversion to open. These findings are noteworthy because body habitus and pelvic operations had been theorized as situations in which the use of the surgical robot would confer an advantage. 
Another comprehensive study of 2,735 patients showed a significantly higher rate of conversion in the laparoscopic compared to robotic group. This was found throughout the general cohort as well as in rectal specific procedures, which had a conversion rate of $7.8 \%$ vs. $21.2 \%$, respectively $(P<0.001)^{[12]}$. While these studies boast large sample sizes, the two arms were unevenly matched in terms of number of patients, which may have affected the results. This issue is common throughout the literature on this topic, given the novelty of robotic surgery. A meta-analysis of four randomized controlled trials (the highest quality evidence available), found a significant difference in conversion rates of patients undergoing colorectal resections for cancer. The robotic procedures had lower rates of conversion to open (1.82\%) vs. laparoscopic procedures $(9.48 \%), P<0.04^{[13]}$.

Upon review of this data, one can safely argue that robotic procedures are associated with lower rates of conversion compared to laparoscopic surgeries. However, it should be noted that there are many confounding factors which may affect these results. Patients with a hostile abdomen, advanced tumours, or other considerations may be planned to undergo laparoscopic instead of robotic surgery if the surgeon is anticipating a high likelihood of conversion. Other scenarios may have presented with unexpected intraoperative findings that would have required the case to be converted no matter which modality was used.

\section{Operative time}

Successful performance of surgery is not measured by the time required to perform the task. However, increased operative time correlates with many adverse perioperative outcomes. Data collected across all surgery specialties demonstrates that operative time was $30 \mathrm{~min}$ longer in patients with surgical site infections than in those without ${ }^{[14]}$. Increased operative time has also been shown to result in higher rates of ileus and length of $\operatorname{stay}^{[15,16]}$.

Advocates for laparoscopic surgery often attribute the time needed to dock the robot as a drawback to robotic surgery. The progress of the surgery comes to a halt during this time, particularly with procedures spanning two abdominal quadrants that require a second docking of the robot. Robot docking times have a reported mean of 3-11 $\mathrm{min}^{[17,18]}$. Longer operative times in robotic compared to laparoscopic surgeries were reported in earlier studies performed between $2010-2014^{[9,12]}$, however, later studies have found no difference in times comparing laparoscopic with robotic colorectal resections ${ }^{[11,19]}$.

Global utilization of the robot has enabled an international meta-analysis of 22 studies comparing operative times, demonstrating a significant difference in favour of laparoscopic over robotic procedures ${ }^{[20]}$. Surgeon experience, case volume, and consistency of the operative team are major factors affecting operative efficiency for both methods. A more detailed analysis of operative time will be discussed below with an overview of the learning curve.

\section{Cost}

Laparoscopic instruments are repeatedly reusable, apart from energy devices and staplers which are discarded after one operation. Robotic instruments are calibrated for a finite number of uses per instrument; typically, after ten operations the instrument must be replaced. However, the individual instruments contribute to only a small part compared to the cost of using the platform. It is difficult to capture cost differences regarding the procedure itself, as many studies utilize the total cost of hospitalization as their endpoint. Alharthi et al. ${ }^{[21]}$ looked at sigmoid colectomies and found a cost difference of $\$ 45,057 \mathrm{vs} . \$ 57,871$ in favour of laparoscopic procedures. This margin has been repeatedly demonstrated on subsequent studies including a meta-analysis of randomized controlled trials, with a reported average higher cost of $\$ 8,000$ $\$ 10,000$ for robotic procedures ${ }^{[22,23]}$. 
A few studies have been successful in evaluating the cost differences between robotic and laparoscopic surgery examining only the charges specific to the procedure. Ramji et al. ${ }^{[24]}$ found the intraoperative robotic costs to be twice as much compared to laparoscopic costs Some evidence exists that increased experience over time leads to fewer charges with robotic procedures. Al-Mazrou et al ${ }^{[7]}$ found that, despite a significantly higher cost with the robot over a span of three years, the cost difference reduced over time for the robotic group: \$2698 in 2012, \$2235 in 2013, and \$1402 in 2014. Given the lack of significant differences in many perioperative parameters between the two groups, the cost associated with the robotic procedure is the one consistent metric upon which improvements can be made.

\section{POSTOPERATIVE OUTCOMES}

\section{Pain}

Robotic and laparoscopic surgery share similar incisions via trocar and specimen extraction sites. A robotic right colectomy typically involves an intracorporeal anastomosis, allowing for extraction through a Pfannenstiel incision at the conclusion of the procedure. Investigating this theory of a less painful incision, Kelley et al. ${ }^{[25]}$ reported a $50 \%$ lower use of postoperative narcotics in the robotic group compared to laparoscopic right hemicolectomies. Within the literature specific to rectal procedures, there are no statistically significant differences in postoperative pain scores or analgesic use between the two interventions ${ }^{[18,26]}$.

\section{lleus}

There were no observed differences in several independent studies of laparoscopic vs. robotic rectal resections in terms of postoperative ileus. Pooled data from eight studies with 854 patients failed to reach significance regarding the incidence of prolonged ileus between the two groups, nor was there a difference in time to resumption of regular diet ${ }^{[27]}$. Feinberg et al. ${ }^{[11]}$ reported ileus rates of $9.5 \%$ for robotic $v s .10 .4 \%$ for laparoscopic rectal resections, which was not statistically significant. While the rates of ileus were as high as $12 \%-17 \%$ in another study, comparisons between laparoscopic and robotic surgery continued to show no difference. Conversely, a meta-analysis of over 125,989 patients undergoing colectomies did show a statistically significant faster time to recovery of bowel function in the robotic group, though these groups were vastly uneven $(121,055 \text { laparoscopic patients } v s .4,934 \text { robotic patients })^{[28]}$. Given the plethora of casematched, well-performed studies listed previously, there is no clear difference in ileus rates between the two methods.

\section{Surgical infection and anastomotic leak}

Surgical site infection and anastomotic leak rates were not shown to be different between the robotic and laparoscopic surgery groups for rectal cancer in the ROLARR randomized clinical trial ${ }^{[29]}$. The results from this international, multi-institutional study support the findings of four other independent studies demonstrating no significant difference between the two arms $s^{[9,11,30,31]}$.

\section{Length of stay}

Multiple studies have shown no difference in the length of stay between robotic and laparoscopic proctectomy $^{[9,32]}$. While two studies claim statistically significant differences in data (0.6 days and 0.4 days, both favouring robotic surgery), this does not translate into clinical relevance ${ }^{[12,33]}$. Additionally, it is also hard to determine if the cost of hospitalization is affected due to the time difference. Many confounding variables can account for differences this diminutive, including wait times while admitted in holding prior to the procedure, operative times, and availability of transportation home on the day of discharge. A comprehensive review of the National Impatient Sample database by Alharthi et al ${ }^{[21]}$ did show a meaningful difference of 4.8 days for robotic vs. 5.7 days for laparoscopic approach. This study is the only one to also look at hospital charges, and despite a shorter length of stay the robotic group still had significantly higher hospital charges. 


\section{Urogenital/sexual function}

Pelvic dissection carries a risk of urogenital dysfunction secondary to nerve injury, particularly during total mesorectal excision. Injury to the hypogastric plexus in the presacral space and the pelvic splanchnic nerves (nervi erigentes) in the pelvic sidewall can cause significant morbidity. Postoperative urinary retention and erectile dysfunction may result from damage to these nerves. Proposed benefits of the robotic platform include more precise dissection afforded by the wristed instruments and better depth perception due to the binocular lens. These two advantages are postulated to help identify and preserve these nerve branches to reduce morbidity. There is consensus in the literature that postoperative urinary function scores in women fail to show a difference between robotic and laparoscopic surgery ${ }^{[29,34,35]}$. The standardized International Prostatic Symptom Score is typically used to measure male urinary function scores. Two prospective studies, as well as a meta-analysis, failed to show a major difference in male urinary symptoms at multiple time points postoperatively when comparing the two surgical techniques ${ }^{[29,34,36,37]}$. Other studies revealed only minor differences regarding an earlier return to baseline function, or shorter time to catheter removal $^{[30,35]}$.

Sexual function returns faster in patients after undergoing robotic procedures $v$ s. traditional laparoscopy. Reviewing quality of life and sexual outcomes in robotic surgery specific to rectal cancer, there was no difference in erectile function comparing high $v s$. low anterior resection $v s$. abdominoperineal resection. Luca et al. ${ }^{[38]}$ examined only robotic procedures and reported a decrease in erectile dysfunction compared to baseline at 3 and 6 months, but returning to baseline in one year. The literature comparing sexual function after robotic $v s$. laparoscopic resection consistently demonstrates favourable outcomes for robotic surgery. A case-matched comparison between laparoscopic and robotic procedures revealed no change from baseline at one year, however the robotic group had significantly better erectile function within the first six months ${ }^{[36]}$. Another comparative study supported these findings which found that male sexual function scores deteriorated across all components of the questionnaire in the laparoscopic group but not in the robotic group ${ }^{[35]}$. Given this review of the literature, urinary outcomes are comparable between the groups, however the data favours robotic surgery for earlier return to baseline sexual function.

\section{Learning curve}

Transitioning from open procedures to minimally invasive techniques can be a daunting task for the surgeon. While both robotic and laparoscopic surgery are an entirely new skill set, it is postulated that the robotic platform facilitates this shift to modern surgical techniques. Patient outcomes and surgical efficiency are standard outcomes for measuring the learning curve for these operations. The literature supports a rather short learning curve for the robotic platform, as many surgeons are already experienced in laparoscopic surgery and quickly make the adjustment to robotics. A systematic review of the learning curve evaluated by operative times found a range of 5 to 310 cases for laparoscopic surgery (most were in the range of 35-50), and 15 to 30 cases for robotic surgery ${ }^{[39]}$. Other studies also support a case volume of 50-80 laparoscopic $v$ s. 20-50 robotic surgeries is necessary to attain proficiency ${ }^{[10,40,41]}$.

A unique study investigated the simultaneous learning curves of a surgical fellow/trainee by evaluating both laparoscopic and robotic right hemicolectomy operative times. The numbers of procedures required to identify a decrease in operative time was determined to be 16 for robotic surgery and 25 for laparoscopic surgery ${ }^{[42]}$. There is evidence that surgeons with little to no laparoscopic experience can successfully transition directly to the robotic platform. Kim et al. ${ }^{[43]}$ compared two surgeons performing 100 rectal cancer cases, one of which had performed less than 30 laparoscopic procedures $v s$. a surgeon that had done over 300 laparoscopic surgeries. The inexperienced minimally invasive surgeon showed a marked decrease in operative time after 17 cases and had shorter operative times at the completion of the study without any difference in oncological outcomes compared to the seasoned laparoscopist. In conclusion, the learning curve for robotic surgery is faster than laparoscopy, and feasible for all experience levels. 


\section{CONCLUSION}

Robotic surgery has gained widespread popularity for many reasons. Ergonomically, many surgeons prefer the robot console for comfort over the course of lengthy operations. Workplace overuse injuries specific to laparoscopic surgery can include neck, lower back, and wrist ailments, due to instrument handling and monitor positioning. The reported percentage of musculoskeletal disorders ranges from $73 \%-100 \%$ for laparoscopic surgery and $23 \%-80 \%$ for robotic surgery ${ }^{[44]}$. Electromyography has been used to compare muscle activation between laparoscopic and robotic surgery, revealing that muscle activation was higher in most muscle groups in laparoscopic compared to robotic surgeons ${ }^{[45]}$. The only muscle group that did not show significant difference in activation was the trapezius, and this was correlated to poor positioning of the robotic eyepiece. Lee et al. ${ }^{[46]}$ surveyed 432 exclusively robotic surgeons and reported that $56 \%$ of surgeons still have discomfort manifested by eye strain, neck stiffness, and finger fatigue, as well as lower back stiffness with increased surgical volumes.

This review of the literature clearly demonstrates both minimally invasive techniques to be equivalent in terms of meaningful perioperative outcomes, though intraoperative costs are consistently higher for robotic surgery. One must be careful when evaluating the data as there are many unmeasurable confounding factors that may affect outcomes. Conversion rates may be misrepresented in favour of robotic surgery if complicated cases with an anticipated high likelihood of conversion to open surgery were planned laparoscopically to save time docking the robot. Operative times may be misrepresented as none of the data evaluated teaching atmospheres and time given to the surgical trainee $v s$. the attending physician. The narrow length of stay differences may be affected by preoperative delays or postoperative transportation availability. Despite all these possibilities, the two platforms continue to show negligible differences that do not reach statistical significance in almost all studies. The robotic platform may boast a shorter learning curve, though it should be noted in many of these cases the surgeon already has laparoscopic experience performing the procedure and understanding the planes of dissection.

Much effort has been spent evaluating the difference between these platforms. However, the incision, extraction site, surgeon, and operation are the same, save for the advanced capabilities inherent in the robotic instruments. The ultimate decision on whether to perform robotic $v s$. laparoscopic surgery should be based on surgeon preference and familiarity with equipment, as well as local resources.

\section{DECLARATIONS}

\section{Authors' contributions}

Manuscript preparation: Kavalukas SL, Ghuman A, Sharp SP

Manuscript review and Editing: Wexner SD

\section{Availability of data and materials}

Not applicable.

\section{Financial support and sponsorship}

None.

\section{Conflicts of interest}

Dr Wexner is a paid consultant and receives royalties for intellectual property license from Intuitive Surgical, Medtronic, and Karl Storz and is a paid consultant for Stryker. all other authors declared that there are no conflicts of interest.

\section{Ethical approval and consent to participate}

Not applicable. 


\section{Consent for publication}

Not applicable.

\section{Copyright}

(c) The Author(s) 2020.

\section{REFERENCES}

1. Jacobs M, Verdeja JC, Goldstein HS. Minimally invasive colon resection (laparoscopic colectomy). Surg Laparosc Endosc1991;1:144-50.

2. Guller U, Jain N, Hervey S, Purves H, Pietrobon R. Laparoscopic vs open colectomy: outcomes comparison based on large nationwide databases. Arch Surg 2003;138:1179-86.

3. Masoomi H, Buchberg B, Nguyen B, Tung V, Stamos MJ, et al. Outcomes of laparoscopic versus open colectomy in elective surgery for diverticulitis. World J Surg 2011;35:2143-8.

4. Klaristenfeld DD, McLemore EC, Li BH, Abbass MA, Abbas MA. Significant reduction in the incidence of small bowel obstruction and ventral hernia after laparoscopic compared to open segmental colorectal resection. Langenbeck's Arch Surg 2015;400:505-12.

5. Wexner SD, Johansen OB. Laparoscopic bowel resection: advantages and limitations. Ann Med 1992;24:105-10.

6. Weber PA, Merola S, Wasielewski A, Ballantyne GH, Delaney CP. Telerobotic-assisted laparoscopic right and sigmoid colectomies for benign disease. Dis Colon Rectum 2002;45:1689-94.

7. Al-Mazrou AM, Baser O, Kiran RP. Propensity score-matched analysis of clinical and financial outcomes after robotic and laparoscopic colorectal resection. J Gastrointest Surg 2018;22:1043-51.

8. Vasudevan V, Reusche R, Wallace H, Kaza S. Clinical outcomes and cost-benefit analysis comparing laparoscopic and robotic colorectal surgeries. Surg Endosc 2016;30:5490-3.

9. Sawada H, Egi H, Hattori M, Suzuki T, Shimomura M, et al. Initial experiences of robotic versus conventional laparoscopic surgery for colorectal cancer, focusing on short-term outcomes: a matched case-control study. World J Surg Oncol 2015;13:1-6.

10. Akmal Y, Bailey C, Baek JH, Metchikian M, Pigazzi A. Oncological outcomes of laparoscopic colon resection for cancer after implementation of a full-time preceptorship. Surg Endosc 2011;25:2967-71.

11. Feinberg AE, Elnahas A, Bashir S, Cleghorn MC, Quereshy FA. Comparison of robotic and laparoscopic colorectal resections with respect to 30-day perioperative morbidity. Can J Surg 2016;59:262-7.

12. Tam MS, Kaoutzanis C, Mullard AJ, Regenbogen SE, Franz MG, et al. A population-based study comparing laparoscopic and robotic outcomes in colorectal surgery. Surg Endosc 2016;30:455-63.

13. Liao G, Zhao Z, Lin S, Li R, Yuan Y, et al. Robotic-assisted versus laparoscopic colorectal surgery: A meta-analysis of four randomized controlled trials. World J Surg Oncol 2014;12:1-11.

14. Cheng H, Chen BPH, Soleas IM, Ferko NC, Cameron CG. Prolonged operative duration increases risk of surgical site infections: a systematic review. Surg Infect (Larchmt) 2017;18:722-35.

15. Harnsberger CR, Maykel JA, Alavi K. Postoperative ileus. Clin Colon Rectal Surg 2019;32:166-70.

16. Harrison OJ, Smart NJ, White P, Brigic A, Carlisle ER, et al. Operative time and outcome of enhanced recovery after surgery after laparoscopic colorectal surgery. J Soc Laparoendosc Surg 2014;18:265-72.

17. Dehal A, Patel S, Park H, Nguyen P, Yuhan R, et al. Robotic colorectal surgery: our initial experience. Am Surg 2016;82:907-10.

18. Shin JY. Comparison of short-term surgical outcomes between a robotic colectomy and a laparoscopic colectomy during early experience. J Korean Soc Coloproctol 2012;28:19-26.

19. Nolan HR, Smith BE, Honaker MD. Operative time and length of stay is similar between robotic assisted and laparoscopic colon and rectal resections. J Robot Surg 2018;12:659-64.

20. Lorenzon L, Bini F, Balducci G, Ferri M, Salvi PF, et al. Laparoscopic versus robotic-assisted colectomy and rectal resection: a systematic review and meta-analysis. Int J Colorectal Dis 2016;31:161-73.

21. Alharthi S, Reilly M, Arishi A, Ahmed AM, Chulkov M, et al. Robotic versus laparoscopic sigmoid colectomy: analysis of healthcare cost and utilization project database. Am Surg 2020;86:256-60.

22. Silva-Velazco J, Dietz DW, Stocchi L, Costedio M, Gorgun E, et al. Considering value in rectal cancer surgery: an analysis of costs and outcomes based on the open, laparoscopic, and robotic approach for proctectomy. Ann Surg2017;265:960-8.

23. Roh HF, Nam SH, Kim JM. Robot-assisted laparoscopic surgery versus conventional laparoscopic surgery in randomized controlled trials: a systematic review and meta-analysis. PLoS One 2018;13:e0191628.

24. Ramji KM, Cleghorn MC, Josse JM, MacNeill A, O’Brien C, et al. Comparison of clinical and economic outcomes between robotic, laparoscopic, and open rectal cancer surgery: early experience at a tertiary care center. Surg Endosc 2016;30:1337-43.

25. Kelley SR, Duchalais E, Larson DW. Short-term outcomes with robotic right colectomy. Am Surg 2018;84:1768-73.

26. Tolstrup R, Funder JA, Lundbech L, Thomassen N, Iversen LH. Perioperative pain after robot-assisted versus laparoscopic rectal resection. Int J Colorectal Dis 2018;33:285-9.

27. Trastulli S, Farinella E, Cirocchi R, Cavaliere D, Avenia N, et al. Robotic resection compared with laparoscopic rectal resection for cancer: systematic review and meta-analysis of short-term outcome. Colorectal Dis 2012;14:e134-56.

28. Chang YS, Wang JX, Chang DW. A meta-analysis of robotic versus laparoscopic colectomy. J Surg Res 2015;195:465-74.

29. Jayne D, Pigazzi A, Marshall H, Croft J, Corrigan N, et al. Effect of robotic-assisted vs conventional laparoscopic surgery on risk of 
conversion to open laparotomy among patients undergoing resection for rectal cancer the rolarr randomized clinical trial. JAMA - J Am Med Assoc 2017;318:1569-80.

30. Ye SP, Zhu WQ, Liu DN, Lei X, Jiang QG, et al. Robotic- vs laparoscopic-assisted proctectomy for locally advanced rectal cancer based on propensity score matching: Short-term outcomes at a colorectal center in China. World J Gastrointest Oncol 2020;12:424-34.

31. Schootman M, Hendren S, Loux T, Ratnapradipa K, Eberth JM, et al. Differences in effectiveness and use of robotic surgery in patients undergoing minimally invasive colectomy. J Gastrointest Surg 2017;21:1296-303.

32. Park JS, Choi GS, Lim KH, Jang YS, Jun SH. Robotic-assisted versus laparoscopic surgery for low rectal cancer: Case-matched analysis of short-term outcomes. Ann Surg Oncol 2010;17:3195-202.

33. Hopkins MB, Geiger TM, Bethurum AJ, Ford MM, Muldoon RL, et al. Comparing pathologic outcomes for robotic versus laparoscopic surgery in rectal cancer resection: a propensity adjusted analysis of 7616 patients. Surg Endosc 2020;34:2613-22.

34. Kim HJ, Choi GS, Park JS, Park SY, Yang CS, et al. The impact of robotic surgery on quality of life, urinary and sexual function following total mesorectal excision for rectal cancer: a propensity score-matched analysis with laparoscopic surgery. Colorectal Dis 2018; 20:0103-13.

35. Panteleimonitis S, Ahmed J, Ramachandra M, Farooq M, Harper M, et al. Urogenital function in robotic vs laparoscopic rectal cancer surgery: a comparative study. Int J Colorectal Dis 2017;32:241-8.

36. Park SY, Choi GS, Park JS, Kim HJ, Ryuk JP, et al. Urinary and erectile function in men after total mesorectal excision by laparoscopic or robot-assisted methods for the treatment of rectal cancer: a case-matched comparison. World J Surg 2014;38:1834-42.

37. Tang X, Wang Z, Wu X, Yang M, Wang D. Robotic versus laparoscopic surgery for rectal cancer in male urogenital function preservation, a meta-analysis. World J Surg Oncol 2018;16:196.

38. Luca F, Valvo M, Ghezzi TL, Zuccaro M, Cenciarelli S, et al. Impact of robotic surgery on sexual and urinary functions after fully robotic nerve-sparing total mesorectal excision for rectal cancer. Ann Surg 2013;257:672-8.

39. Barrie J, Jayne DG, Wright J, Murray CJC, Collinson FJ, et al. Attaining surgical competency and its implications in surgical clinical trial design: a systematic review of the learning curve in laparoscopic and robot-assisted laparoscopic colorectal cancer surgery. Ann Surg Oncol 2014;21:829-40.

40. Lee JM, Yang SY, Han YD, Cho MS, Hur H, et al. Can better surgical outcomes be obtained in the learning process of robotic rectal cancer surgery? A propensity score-matched comparison between learning phases. Surg Endosc 2020; doi: 10.1007/s00464-020-07445-3.

41. Park EJ, Kim CW, Cho MS, Kim DW, Min BS, et al. Is the learning curve of robotic low anterior resection shorter than laparoscopic low anterior resection for rectal cancer?: a comparative analysis of clinicopathologic outcomes between robotic and laparoscopic surgeries. Med (United States) 2014;93:1-11.

42. De'Angelis N, Lizzi V, Azoulay D, Brunetti F. Robotic versus laparoscopic right colectomy for colon cancer: analysis of the initial simultaneous learning curve of a surgical fellow. J Laparoendosc Adv Surg Tech 2016;26:882-92.

43. Kim IK, Kang J, Park YA, Kim NK, Sohn SK, et al. Is prior laparoscopy experience required for adaptation to robotic rectal surgery?: Feasibility of one-step transition from open to robotic surgery. Int J Colorectal Dis 2014;29:693-9.

44. Catanzarite T, Tan-Kim J, Whitcomb EL, Menefee S. Ergonomics in surgery: a review. Female Pelvic Med Reconstr Surg 2018;24:1-12.

45. Zárate Rodriguez JG, Zihni AM, Ohu I, Cavallo JA, Ray S, et al. Ergonomic analysis of laparoscopic and robotic surgical task performance at various experience levels. Surg Endosc 2019;33:1938-43.

46. Lee GI, Lee MR, Green I, Allaf M, Marohn MR. Surgeons' physical discomfort and symptoms during robotic surgery: a comprehensive ergonomic survey study. Surg Endosc 2017;31:1697-706. 